\title{
Pump dependent carrier lifetimes in InAs/ GaAs quantum dot photoconductive terahertz antenna structures
}

Cite as: J. Appl. Phys. 125, 151606 (2019); https://doi.org/10.1063/1.5083798

Submitted: 30 November 2018 . Accepted: 15 March 2019 . Published Online: 01 April 2019

Andrei Gorodetsky (D), Natalia Bazieva, and Edik U. Rafailov (D)

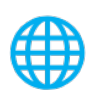

View Online

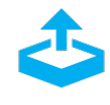

Export Citation

\section{ARTICLES YOU MAY BE INTERESTED IN}

Enhanced terahertz emission from strain-induced InGaAs/InAlAs superlattices Journal of Applied Physics 125, 151605 (2019); https://doi.org/10.1063/1.5079697

Wavelength-selective enhancement of terahertz absorption of metallic grating/GaAs-based hybrid photoconductive detector

Journal of Applied Physics 125, 151604 (2019); https://doi.org/10.1063/1.5085449

High-sensitivity photoconductive detectors with wide dipole electrodes for low frequency THz wave detection

Journal of Applied Physics 125, 151610 (2019); https://doi.org/10.1063/1.5080750 


\title{
Pump dependent carrier lifetimes in InAs/GaAs quantum dot photoconductive terahertz antenna structures
}

\author{
Cite as: J. Appl. Phys. 125, 151606 (2019); doi: 10.1063/1.5083798 \\ Submitted: 30 November 2018 - Accepted: 15 March 2019 . \\ Published Online: 1 April 2019

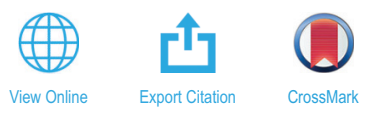

Andrei Gorodetsky, ${ }^{1,2, a)}$ (D) Natalia Bazieva, ${ }^{3}$ and Edik U. Rafailov ${ }^{3}$ (iD

\author{
AFFILIATIONS \\ ${ }^{7}$ Faculty of Physics and Engineering, ITMO University, St. Petersburg 197101, Russia \\ ${ }^{2}$ Department of Chemistry, Imperial College London, London SW7 2AZ, United Kingdom \\ ${ }^{3}$ Optoelectronics and Biomedical Photonics Group, AIPT, Aston University, Birmingham B4 7ET, United Kingdom
}

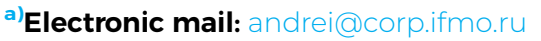

\begin{abstract}
In this paper, we study the behavior of photoexcited carriers in GaAs wafers containing self-assembled InAs quantum dots (QDs). These samples were designed and grown to be employed as photoconductive antennae for terahertz (THz) generation. The implanted QDs serve to shorten the overall carrier lifetime and enable pumping with lasers operating at wavelengths between $1 \mu \mathrm{m}$ and $1.3 \mu \mathrm{m}$. Optical pump-THz probe measurements reveal carrier lifetime shortening for higher pump powers in the unbiased antennae due to increased carrier capture via Auger relaxation. This phenomenon significantly broadens the THz spectra from QD-based PCAs and explains the increased THz intensity at higher frequencies presented earlier.
\end{abstract}

\section{Published under license by AIP Publishing. https://doi.org/10.1063/1.5083798}

\section{INTRODUCTION}

Self-assembled InAs quantum dots (QDs) in GaAs lattices are widely used in semiconductor photonics as gain media in diode lasers, ${ }^{1}$ amplifiers, ${ }^{2}$ and as saturable absorber mirrors (SESAMs) ${ }^{3}$ that allow subpicosecond pulsed lasing at rather high powers. ${ }^{4}$ Recently, QD materials, specifically designed for the purpose, were demonstrated to successfully generate coherent terahertz (THz) radiation in pulsed ${ }^{5-7}$ and continuous wave $(\mathrm{CW})^{6,8,9}$ regimes after being pumped with compact semiconductor QD-based laser diodes and other sources.

There is a wealth of research in the area of $\mathrm{THz}$ emitters, resulting in several established approaches. To avoid reciting them all here, one can be addressed to well-presented reviews ${ }^{10,11}$ and books. ${ }^{12,13}$ Among all of the generation methods, the best candidates for compact and room-temperature operating sources are on-chip nonlinear semiconductor mixers of mid-IR quantum cascade laser (QCL) radiation $^{14}$ and photoconductive antennae (PCAs) ${ }^{11}$ that can be pumped by compact ultrafast fiber ${ }^{15}$ and semiconductor ${ }^{1,4}$ lasers. Most of these compact lasers operate at energies below the bandgap of the most popular ultrafast material, low-temperature grown GaAs
(LT-GaAs). To make such compact systems viable, several approaches have been developed, including the use of LT-InGaAs/InAlAs multilayer structures, ${ }^{16}$ GaBiAs-based substrates, ${ }^{17}$ two-photon absorption enhancement with plasmonic nanostructures, ${ }^{18}$ and the use of the above mentioned QD-based photoconductive devices. ${ }^{6,9}$

For efficient $\mathrm{THz}$ generation, short carrier lifetimes are an important material property for PCAs in both the pulsed and CW pumping conditions. ${ }^{10,11,19}$ Because the THz far field time domain electric field amplitude is proportional to the time derivative of the photocurrent inside the photogap of the PCA or photomixer (PM), ${ }^{11}$ longer carrier lifetimes lead not only to the narrowing of the resulting $\mathrm{THz}$ spectrum, but also to a decrease in the overall intensity of the generated $\mathrm{THz}$ signal. Traditionally, these issues have been circumvented by employing low-temperature grown semiconductors, where defects induced by the growth conditions act as carrier capture sites that shorten the carrier lifetimes. ${ }^{20}$ However, these defects strongly affect the wafer quality and hence reduce the carrier mobility, thermal conductivity, and breakdown threshold and increase nonradiative absorption that does not allow high pump intensities. 
One promising material system for PCA applications is GaAs wafers containing self-assembled InAs QDs. The InAs QDs serve as fast carrier capture sites without compromising the wafer quality of the high-mobility GaAs. InAs/GaAs QD SESAM structures have been shown to have carrier lifetimes below $1 \mathrm{ps}^{3}$. The carrier lifetimes and photoconductive response of QD photoconductive wafers can be engineered at the design stage, thus enabling pump wavelengths beyond $\sim 1 \mu \mathrm{m}^{21}$ to avoid the need for expensive and bulky Ti:Sapphire lasers that are most widely used as pump sources in $\mathrm{THz}$ photonic setups. ${ }^{22}$ To optimally configure the QD material for efficient $\mathrm{THz}$ generation, one needs to study the connection between its structure and properties.

Recently, after the wavelength-dependent $\mathrm{THz}$ generation efficiency was revealed, ${ }^{6}$ we investigated the processes leading to the photoconductivity in these QD substrates. ${ }^{21}$ Our previous experiments on $\mathrm{THz}$ generation showed that the spectral properties of the QD PCA strongly depended on the pump power. ${ }^{6,23}$ Here, we elucidate the reason for superior generation efficiency in the high frequency range by studying the lifetime of the photogenerated carriers in the QD semiconductor wafers with time-resolved optical pump-THz probe spectroscopy. ${ }^{24}$ The results presented herein for carrier lifetime shortening in unbiased QD wafers can be discriminated from the spectral broadening via carrier screening reported earlier. ${ }^{25}$

\section{EXPERIMENTAL RESULTS}

\section{A. Sample preparation}

In this work, two designs of the InAs/GaAs QD wafers for $\mathrm{THz}$ generation were studied, which differ only in the number of QD layers inside: 25 for Structure 1 and 40 for Structure 2. The samples with different layer counts were made to check for any compromise between the wafer quality and the photoconductive efficiency of the resulting PCAs. The samples were fabricated at different sites, but the growth conditions were kept as similar as possible. The structures, depicted generally in Fig. 1(a), were grown by molecular beam epitaxy (MBE) in the Stranski-Krastanov regime on a semi-insulating (SI) GaAs substrate. QDs are set in layers, and their sizes are precalculated to have ground, first, and second excited states at 1260,1180, and $1111 \mathrm{~nm}$, respectively.

To ensure the uniformity of the QD layers in the stack, each QD layer was grown by deposition of 2.3 monolayer (ML) InAs at $500{ }^{\circ} \mathrm{C}$, capped by $4 \mathrm{~nm} \operatorname{In}_{0.15} \mathrm{Ga}_{0.85} \mathrm{As}$, and then by $6 \mathrm{~nm}$ GaAs. The temperature was then raised to $580^{\circ} \mathrm{C}$ before growth of the subsequent $30 \mathrm{~nm}$ GaAs spacer layer in order to desorb segregated In. Also, prior to the growth of each QD layer, the GaAs surface was annealed under an $\mathrm{As}_{2}$ flux for 5 min to flatten the growth surface. AFM analysis gives an estimated QD density of around $3 \times 10^{10} \mathrm{~cm}^{-2}$ per layer. A TEM image of a single QD is shown in the inset of Fig. 1(a). More detailed sample parameters can be found in Ref. 6 .

The operating wavelengths of the samples were selected to work with compact semiconductor laser pumps, which were previously used to demonstrate efficient $\mathrm{CW} \mathrm{THz} \mathrm{operation} \mathrm{of} \mathrm{the} \mathrm{PCAs.}{ }^{9}$ Both structures have a $30 \mathrm{~nm}$ top layer of LT-GaAs to reduce the dark resistance and allow ohmic contact with the metallic PCA electrodes. This layer is very thin compared to the overall thickness of the active regions ( $\sim 1 \mu \mathrm{m}$ for Structure 1 and $\sim 1.4 \mu \mathrm{m}$ for Structure 2 ), and
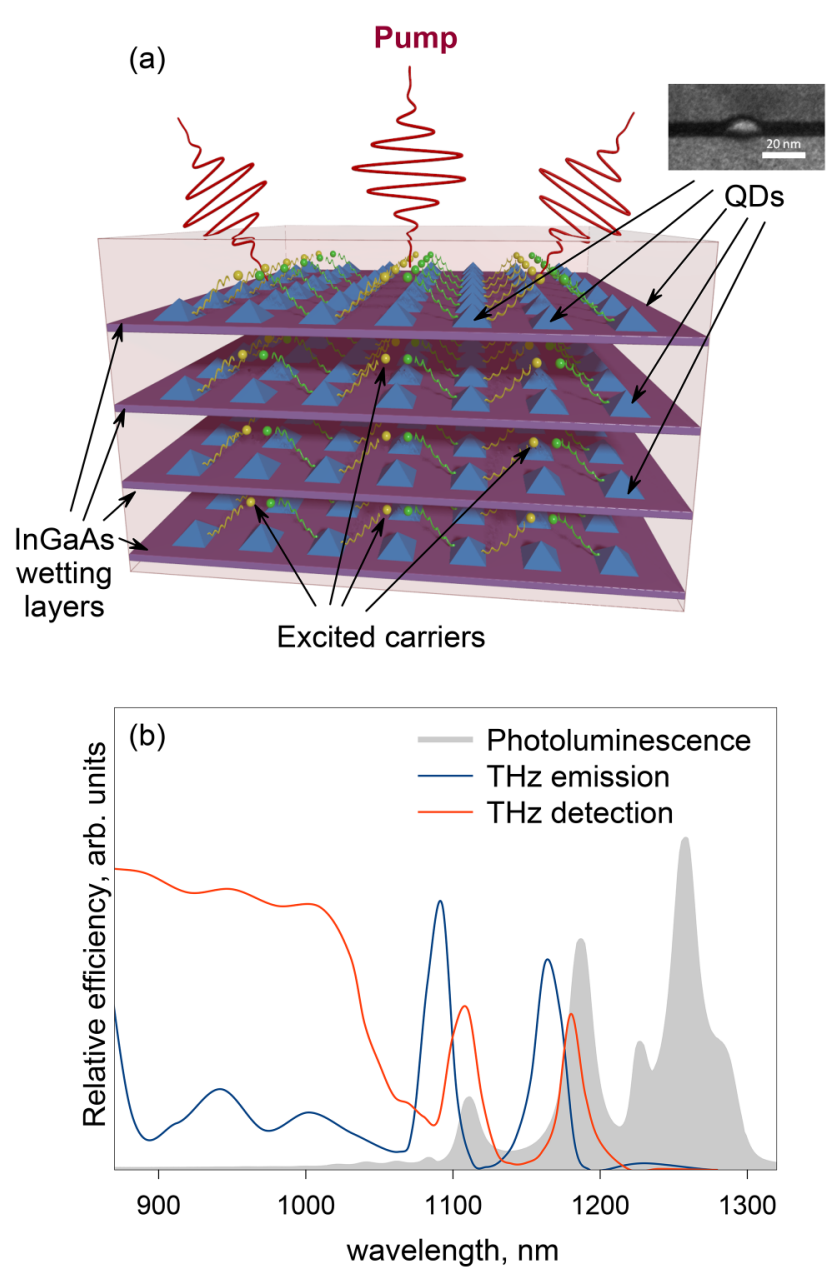

FIG. 1. (a) Illustration of the QD wafer structure. The inset shows the TEM image of a single QD; (b) photoluminescence of the QD sample and its operation as a THz emitter and detector.

should therefore not significantly affect the thermal conductivity or the carrier lifetimes of the samples. The photoluminescence (PL) spectra and pump wavelength dependences of $\mathrm{THz}$ generation and detection efficiency of Structure 2 are shown in Fig. 1(b). ${ }^{6}$ The absence of any appreciable $\mathrm{THz}$ emission under ground state (GS) pumping, despite the strong PL at these energies, has been explained by a slower thermal extraction of generated carriers, in comparison to significantly faster tunneling of the "hot" carriers that are excited at shorter wavelengths, corresponding to the QD excited states (ESs). ${ }^{21}$

\section{B. Experimental setup}

To study the carrier relaxation processes, the samples were pumped with energy above the GaAs bandgap to excite carriers in the whole structure volume. For lifetime estimation, we used a 
time-resolved $\mathrm{THz}$ spectroscopic (IR pump-THz probe) setup ${ }^{24}$ based on a THz time-domain spectrometer (THz-TDS) with the third optical beam independently delayed and used to pump the sample. The setup is shown in Fig. 2.

A Sprite-XT (M Squared Ltd.) femtosecond Ti:sapphire laser was used to generate $800 \mathrm{~nm}$ pulses of $120 \mathrm{fs}$ duration at a repetition rate of $80 \mathrm{MHz}$ and a mean power of $1.85 \mathrm{~W}$. $95 \%$ of the power was sent to pump the sample located in the focal spot of the $\mathrm{THz}$ probe, which in turn was powered by the remaining $5 \%$ of laser power split into two paths. As THz source and detector in the THz-TDS setup, we use Teravil LT-GaAs PCAs. The pump beam is attenuated by crossed polarizers, and independently delayed to allow probing with the $\mathrm{THz}$ pulse at different times after excitation.

$\mathrm{THz}$ transmittance of the wafer is inversely proportional to the number of free charge carriers existing therein, so by varying the delay between the IR pump and $\mathrm{THz}$ probe, we can trace the free carrier dynamics. Typically, and our TDS setup is no exception, the $\mathrm{THz}$ pulse consists of 1-1.5 field oscillations. Also, because we use an unamplified laser source, the signal-to-noise ratio was not optimal in our setup. Therefore, for the sake of comparing the carrier dynamics, we measured the amplitude at the maximum of the $\mathrm{THz}$ pulse.

\section{Lifetime measurement}

Our THz-TDS setup allows focusing into a spot as small as $1 \mathrm{~mm}$ in diameter without a significant loss of signal. The IR pump beam was kept at a comparable size to maximize the effect of transmission change within the overlap. For the maximum pump power, and a pulse repetition rate and Gaussian beam profile of $1 \mathrm{~mm}$ in diameter, the maximum energy density at the semiconductor surface was $J \approx 60 \mu \mathrm{J} / \mathrm{cm}^{2}$. This energy density is comparable to the one achieved at the QD PCA THz generation operation revealing the spectral broadening. ${ }^{6}$ Nevertheless, even at

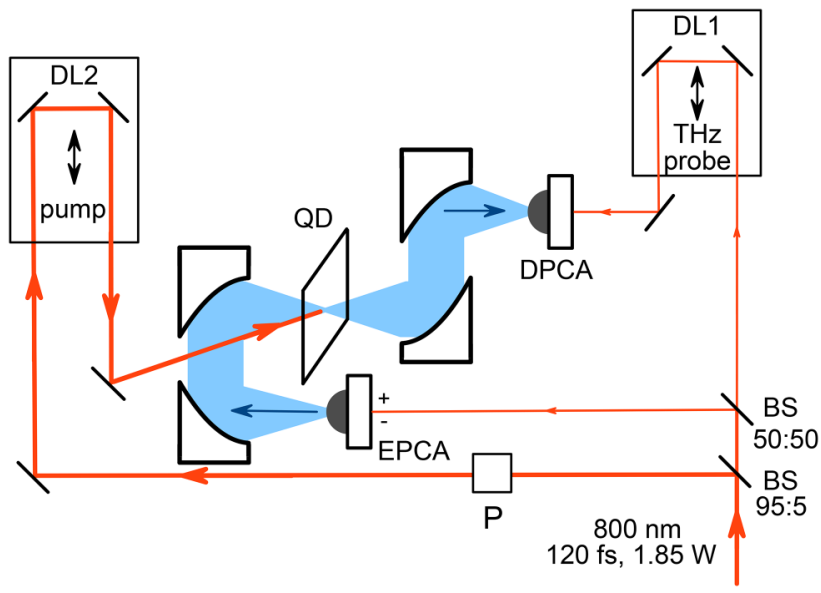

FIG. 2. Experimental setup for IR pump-THz probe measurements. BS—beamsplitters, EPCA—emitter PCA, DPCA—detector PCA, QD—InAs/GaAs QD sample under study, DL1-THz TDS probe delay line, DL2-pump delay line, P-polarizer. this pump level, picosecond dynamics in both QD structures are clearly observed. All scans were performed several times and averaged for enhanced signal-to-noise ratio. Figure 3 shows the power dependence of the differential transmission $-\frac{\Delta T}{T}=\frac{T_{0}-T_{\text {pump }}}{T_{0}}$ vs the pump delay. We emphasize that the $\mathrm{THz}$ radiation probes the concentration of only the "free" carriers present in the GaAs wafer and InGaAs wetting layer (WL), but not the excited carriers captured into QDs, unlike time-resolved PL, or optical pump-probe measurements. Similarly, only the probed mobile electrons are responsible for the $\mathrm{THz}$ generation in the PCA.

As one would expect, higher pump intensities induce a larger change in the transparency of the sample due to the increased concentration of charge carriers. More interestingly, the dynamics of the
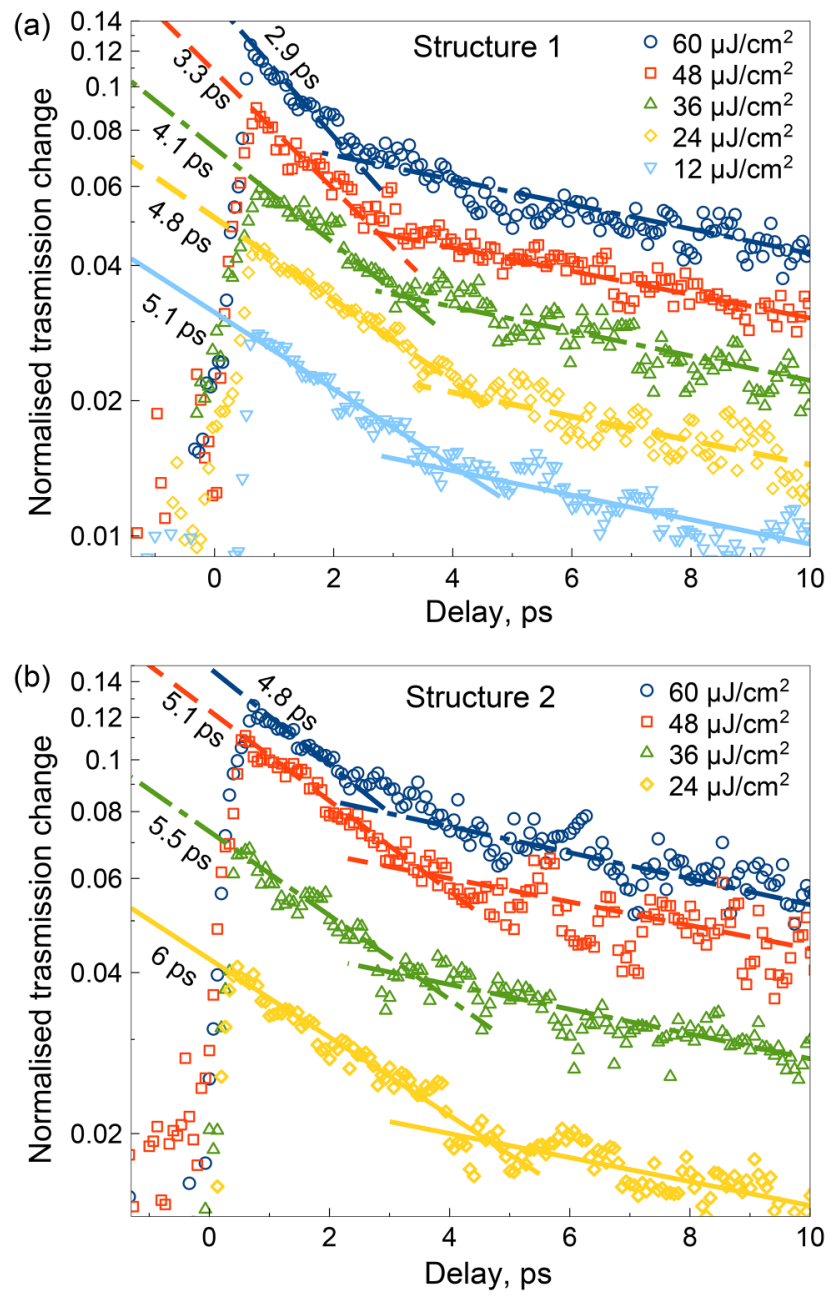

FIG. 3. Relative transmission at maximum $\mathrm{THz}$ pulse amplitude through Structure 1 (a) and Structure 2 (b). Exponential fits are plotted as a guide to the eye and reveal a slower decay component, similar for all pump powers, and gradually shortening with pump power growth faster decay. Numbers show the exponent used for plotting the lines. 
differential transmission curves are clearly composed of two processes with different decay times. We outline these slopes with independent single exponential fits to illustrate this effect more clearly and to estimate the time constants for each process. In both samples, the shallow exponential slope at later times appears to be independent of the pump power, while the steeper slope at earlier times decays more quickly at higher pump powers. The lifetimes range from $\sim 5 \mathrm{ps}$ down to $\sim 3$ ps in Structure 1 and from $\sim 6$ ps down to below 5 ps in Structure 2 when the samples are pumped with $12 \mu \mathrm{J} / \mathrm{cm}^{2}$ to $60 \mu \mathrm{J} / \mathrm{cm}^{2}$ pump energy densities. These lifetimes are much longer than typical values for Auger processes, but the pump intensities used in the measurement suggest that Auger effects might be prominent here. ${ }^{26,27}$ Overall, Structure 1 exhibits shorter lifetimes, which are more dependent on the pump intensity. This might be attributed to the better wafer quality due to a lower number of QD layers and/ or better growth environment. To explain the effect of the lifetime shortening, we turn to a description of the processes involved.

\section{DISCUSSION}

When pumping at $\lambda<850 \mathrm{~nm}$, carriers in the whole wafer volume are excited, and the time scales of nonradiative processes can range from below one to hundreds of picoseconds. ${ }^{27,28}$ These time scales are predominantly determined by the carrier relaxation processes. Time resolved $\mathrm{THz}$ spectroscopy distinguishes the dynamics of carriers in the conductive GaAs substrate from the nonconductive QDs. Hole dynamics, due to their low mobility, are barely detected by the $\mathrm{THz}$ probe. Thus, the measured change in the $\mathrm{THz}$ transmission follows the changes in the free electron induced conductivity of the sample. Therefore, from this point, while using the word "carriers" we refer to electrons only.

The longest living carriers recombine nonradiatively in the low-defect SI GaAs bulk lattice. Two other distinct relaxation times are associated with carrier capture by the QDs, removing carriers from the conductive layers and reducing overall substrate conductivity. The measured carrier capture times are comparable to the ones reported in Ref. 27 measured by ultrafast scanning tunneling microscopy (STM) and induced reflectivity measurements. Auger processes that dominate the carrier capture into QDs in our samples ${ }^{6}$ tend to have shorter times at higher fluences as the Auger carrier-capture time decreases rapidly with increasing fluence. ${ }^{27}$ We separate this ultrafast relaxation component of the Auger capture into QDs at higher fluence into the third, ultrafast relaxation.

To account for the observation of three decay processes, we describe our data with a triexponential model (see Ref. 27). Differential transmission of the $\mathrm{THz}$ probe pulse is proportional to the number of free carriers, $-\frac{\Delta T}{T} \propto N$, and the number of these carriers can be written as

$$
\begin{aligned}
N(t)= & N_{\mathrm{GaAs}} \exp \left(-\frac{t}{\tau_{\mathrm{GaAs}}}\right) \\
& +N_{\mathrm{QD}} \exp \left(-\frac{t}{\tau_{\mathrm{QD}}}\right) \\
& +N_{\mathrm{UA}} \exp \left(-\frac{t}{\tau_{\mathrm{UA}}}\right),
\end{aligned}
$$

where $N_{\mathrm{GaAs}}$ is the number of the carriers that are recombining in the GaAs lattice, $N_{\mathrm{QD}}$ corresponds to the carriers that are trapped into QDs, and $N_{\mathrm{UA}}$ are the carriers that are recombining due to ultrafast Auger processes, respectively. $\tau_{\mathrm{GaAs}}, \tau_{\mathrm{QD}}$, and $\tau_{\mathrm{UA}}$ are the corresponding capture or recombination times.

For the GaAs layers grown at $580^{\circ} \mathrm{C}$, the lattice recombination constant $\tau_{\mathrm{GaAs}} \approx 1 \mathrm{~ns}$ to $2 \mathrm{~ns}^{27,29}$ To derive $\tau_{\mathrm{QD}}$, we address the lowest intensity pump scans, since there are substantially fewer carriers in this case, and Auger processes are much less likely. By setting $N_{\mathrm{UA}}=0$, we derive $\tau_{\mathrm{QD}}$ to be $\tau_{\mathrm{QD}}=3$ ps in Structure 1 and $\tau_{\mathrm{QD}}=3.2 \mathrm{ps}$ in Structure 2. After fitting the time-resolved transmission curves for the higher intensity pumps, the carrier recombination time through the excited dot state $\tau_{\mathrm{UA}} \approx 650 \mathrm{fs}$ in Structure 1 and $\tau_{\mathrm{UA}} \approx 800 \mathrm{fs}$ in Structure 2 , which are in very close agreement with the previously measured values in similar samples. ${ }^{3,2}$

Results of this triexponential fitting for the samples under study are shown in Fig. 4. Plots (a) and (b) show $N(t)$ curves for different pump energy densities for Structure 1 and Structure 2, respectively. Plots (c) and (d) present the dependence of the number of carriers split by their capture sites $N(0)=N_{\mathrm{GaAs}}+$ $N_{\mathrm{QD}}+N_{\mathrm{UA}}$ on pump energy density for Structure 1 and Structure 2 , respectively. The values for $N_{\mathrm{GaAs}}, N_{\mathrm{QD}}$, and $N_{\mathrm{UA}}$ were derived from the retrieved parameters in the fitting.

In both structures, the number of carriers recombining slowly into the lattice grows with the pump power, whereas the number of carriers captured with the QDs remains relatively constant. Under higher pump intensities, the carriers concentration becomes high enough to engage recombination through ultrafast Auger processes. As a result, even though the number of carriers that recombine in the bulk GaAs lattice grows with the pump fluence, the effective lifetime of the carriers in the wafer decreases.

Previously, in similar structures, carrier lifetime shortening $3,27,30$ was reported for resonant pumping, i.e., pump wavelengths corresponding to QD energy bands, where carriers are excited inside the dots and subsequently extracted, ${ }^{21}$ and off-resonant pumping, where the photon energy exceeds the GaAs bandgap, and photocarriers are generated in the bulk of GaAs. On the other hand, elongation of the carrier lifetimes $^{31-33}$ at resonant pump wavelength was observed for higher pump powers.

Our earlier works have shown that QD-based antennae can successfully generate $\mathrm{THz}$ radiation in pulsed ${ }^{5-7}$ and $\mathrm{CW}^{6,8,9}$ regimes, and also revealed a temporal shortening and corresponding spectral broadening of the generated $\mathrm{THz}$ radiation with increasing pump intensity. ${ }^{6,34}$ Previously, similar shortening of the generated $\mathrm{THz}$ pulses was observed in bulk LT-GaAs-based PCA and was ascribed to a carrier screening effect; ${ }^{25}$ however, the rate of change in the case of QD-based PCAs was higher.

To discriminate these two effects, we performed a simulation of the photocurrent in the PCA using the carrier lifetimes derived herein and compare the spectra with those experimentally observed in Ref. 6. The simulation approach is well described in Refs. 35 and 36. In the case of the QD-based substrate, we split the total number of photogenerated carriers into three uneven parts with proportions and time constants derived from the above mentioned triexponential carrier concentration model, before numerical integration. Also, we omitted the carrier screening effect in the model to discriminate the impact of lifetime shortening. After applying a Fourier transform to time 

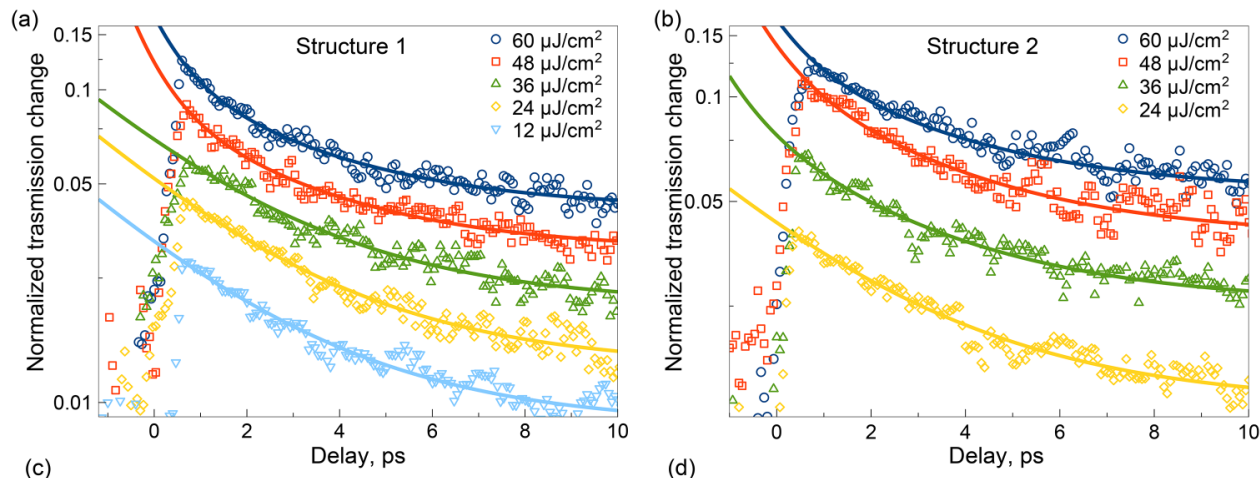

(d)

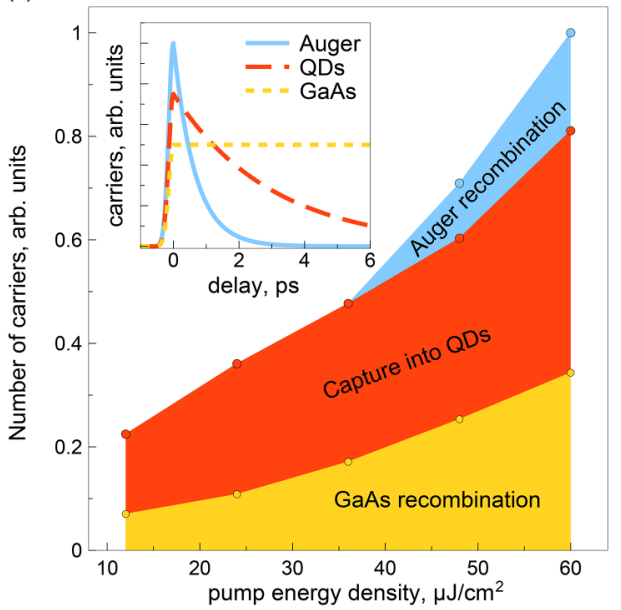

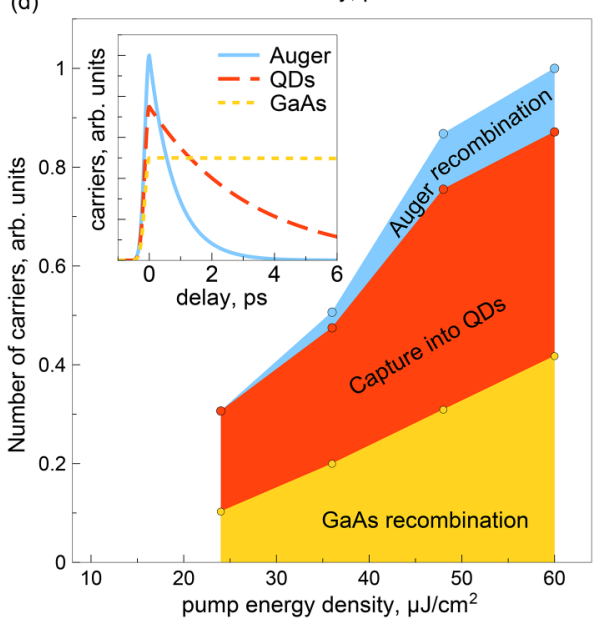

FIG. 4. Differential transmission of $\mathrm{THz}$ radiation at maximum $\mathrm{THz}$ pulse amplitude through Structure 1 (a) and Structure $2(b)$ fulfilled by triexponential approximation. Relative number of carriers relaxing through different capture and relaxation sites derived from triexponent approximation for Structure 1 (c) and Structure 2 (d). Insets show sites' lifetimes used in approximation.

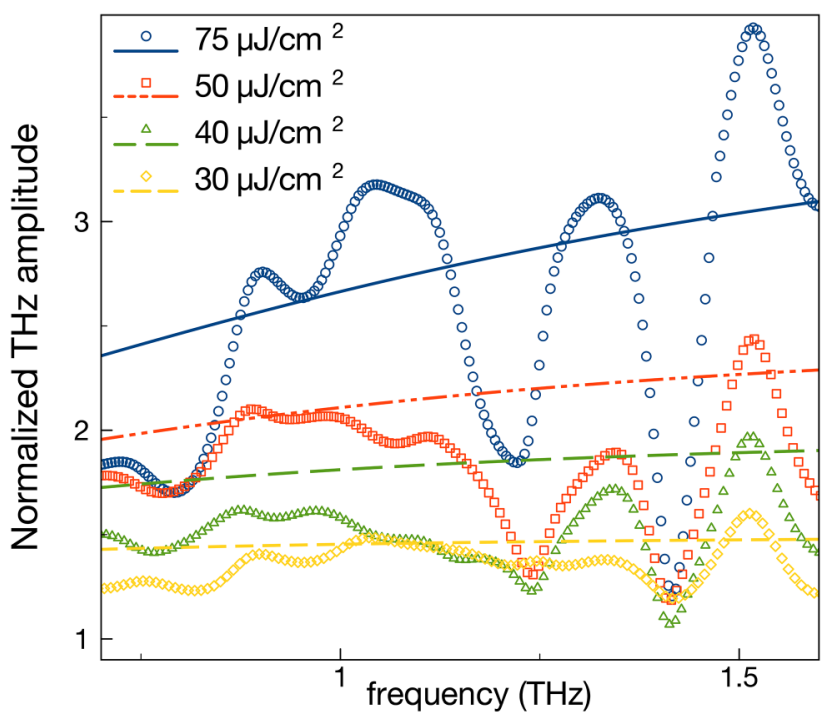

FIG. 5. Normalized THz signal from QD PCA at different pump intensities. Open markers show experimental data and lines depict simulation results. dependent $\mathrm{THz}$ field, the resulting spectra are normalized to one at $20 \mu \mathrm{J} / \mathrm{cm}^{2}$ to correspond to the conditions used in Ref. 6 .

This comparison between simulation and experimental data is presented in Fig. 5. Open markers show the experimental results and the corresponding colored lines show the simulation result. At lower pump powers, the normalized spectra are quite flat, meaning that only the amplitude, but not the energy distribution of the generated spectrum depends on the pump power. On the other hand, for the highest pump power at $75 \mu \mathrm{J} / \mathrm{cm}^{2}$, the normalized spectrum reveals a clear slope that represents an uneven spectral intensity growth, with stronger gain at higher frequencies $(1 \mathrm{THz}-1.5 \mathrm{THz})$.

As can be seen from Fig. 5, the trend of spectral broadening of the generated $\mathrm{THz}$ signal under higher pump powers experimentally observed earlier ${ }^{6}$ is followed by the spectra, simulated with corresponding input pump powers and carrier lifetimes measured in this work. Sharp features in the experimental curves originate from experimental conditions, such as water vapor absorption in the $\mathrm{THz}$ path, minor imperfections in alignment, or the impact of the antenna detector. The simulation does not take into account all the aforementioned effects, hence the simulated curves look more smooth, while clearly repeating the general trend.

Thus, spectral broadening of the $\mathrm{THz}$ signal from QD-based $\mathrm{THz}$ PCA under off-resonant pumping at higher pump powers ${ }^{6}$ can 
originate not only from screening effect but also from carrier lifetime shortening. Since carrier lifetime is an important parameter that affects the $\mathrm{THz}$ generation efficiency, there is a need for more thorough study and qualitative and quantitative analyses of $\mathrm{THz}$ photocurrent generation in QD-based wafers, because high quality QD wafers are one of the most promising candidates for $\mathrm{THz}$ generation under high pump powers due to their quality and thermal tolerance.

\section{CONCLUSION}

In this paper, ultrashort photocarrier lifetimes in InAs/GaAs QD wafers for $\mathrm{THz}$ generation were revealed by IR pump- $\mathrm{THz}$ probe spectroscopy. The overall carrier lifetimes drop with the intensity of the pump, and differential transmission profiles reveal several relaxation processes with different time scales. These processes are quantitatively assessed by a triexponential model that accounts for different relaxation processes in the wafer; the slowest is associated with the GaAs lattice, while the fastest occurs in the QDs. The results are in good agreement with previous observations, ${ }^{3,27}$ and the lifetime shortening can explain earlier reports of $\mathrm{THz}$ spectral broadening in QD-based materials. ${ }^{6}$ This lifetime shortening opens up a pathway to develop more efficient and broadband QD THz sources in conjunction with high-power semiconductor laser pumps.

\section{ACKNOWLEDGMENTS}

E.U.R. acknowledges support from Engineering and Physical Sciences Research Council (EPSRC) (Grant No. EP/R024898/1) and the Russian Science Foundation (Grant No. 18-15-00172). A.G. acknowledges support from the Russian Foundation for Basic Research (Research Project No. 18-07-01492a). The authors thank Dr. Edmund Clarke and Dr. Daniil Livshits for the sample growth; Mr. Thomas R. Hopper, Dr. Artemii Dmitriev, Dr. Alexander Krasnok, and Dr. Ivo Leite for fruitful discussions; A.G. thanks Magicplot Ltd. for providing a copy of MagicPlot Pro crossplatform plotting and fitting software that was used for preparation of all the figures in this paper.

\section{REFERENCES}

${ }^{1}$ E. U. Rafailov, M. A. Cataluna, and W. Sibbett, Nat. Photonics 1, 395 (2007).

${ }^{2}$ E. U. Rafailov, P. Loza-Alvarez, W. Sibbett, G. S. Sokolovskii, D. A. Livshits, A. E. Zhukov, and V. M. Ustinov, IEEE Photon. Technol. Lett. 15, 1023 (2003).

${ }^{3}$ E. U. Rafailov, S. J. White, A. A. Lagatsky, A. Miller, W. Sibbett, D. A. Livshits, A. E. Zhukov, and V. M. Ustinov, IEEE Photon. Technol. Lett. 16, 2439 (2004).

${ }^{4}$ M. A. Gaafar, A. Rahimi-Iman, K. A. Fedorova, W. Stolz, E. U. Rafailov, and M. Koch, Adv. Opt. Photonics 8, 370 (2016).

${ }^{5}$ A. Gorodetsky, N. Bazieva, and E. U. Rafailov, Proc. SPIE 9737, 97370C (2016).

${ }^{6}$ R. R. Leyman, A. Gorodetsky, N. Bazieva, G. Molis, A. Krotkus, E. Clarke, and E. U. Rafailov, Laser Photon. Rev. 10, 772 (2016).

${ }^{7}$ S. Lepeshov, A. Gorodetsky, A. Krasnok, N. Toropov, T. A. Vartanyan, P. Belov, A. Alú, and E. U. Rafailov, Sci. Rep. 8, 6624 (2018).

${ }^{8}$ T. Kruczek, R. Leyman, D. Carnegie, N. Bazieva, G. Erbert, S. Schulz, C. Reardon, and E. U. Rafailov, Appl. Phys. Lett. 101, 081114 (2012).

${ }^{9}$ K. A. Fedorova, A. Gorodetsky, and E. U. Rafailov, IEEE J. Sel. Top. Quantum Electron. 23, 1 (2017).
${ }^{10} \mathrm{P}$. Jepsen, D. Cooke, and M. Koch, Laser Photon. Rev. 5, 124 (2011).

${ }^{11}$ S. Lepeshov, A. Gorodetsky, A. Krasnok, E. Rafailov, and P. Belov, Laser Photon. Rev. 11, 1600199 (2017).

${ }^{12}$ Y. S. Lee, Principles of Terahertz Science and Technology (Springer US, Boston, 2009), pp. 1-340.

${ }^{13}$ X.-C. Zhang and J. Xu, Introduction to THz Wave Photonics (Springer, 2010), Vol. 29.

${ }^{14}$ M. A. Belkin and F. Capasso, Phys. Scr. 90, 118002 (2015).

${ }^{15}$ M. E. Fermann and I. Hartl, Nat. Photonics 7, 868 (2013).

${ }^{16}$ B. Sartorius, H. Roehle, H. Künzel, J. Böttcher, M. Schlak, D. Stanze, H. Venghaus, and M. Schell, Opt. Express 16, 9565 (2008).

${ }^{17}$ K. Bertulis, A. Krotkus, G. Aleksejenko, V. Pacebutas, R. Adomavicius, G. Molis, and S. Marcinkevicius, Appl. Phys. Lett. 88, 201112 (2006).

${ }^{18}$ A. Jooshesh, V. Bahrami-Yekta, J. Zhang, T. Tiedje, T. E. Darcie, and R. Gordon, Nano Lett. 15, 8306 (2015).

${ }^{19}$ A. Krotkus, J. Phys. D Appl. Phys. 43, 273001 (2010).

${ }^{20}$ A. Krotkus and J.-L. Coutaz, Semicond. Sci. Technol. 20, S142 (2005).

${ }^{21}$ A. Gorodetsky, A. Yadav, E. Avrutin, K. A. Fedorova, and E. U. Rafailov, IEEE J. Sel. Top. Quantum Electron. 24, 1 (2018).

${ }^{22}$ V. G. Bespalov, A. A. Gorodetskiü, I. Y. Denisyuk, S. A. Kozlov, V. N. Krylov, G. V. Lukomski, N. V. Petrov, and S. É. Putilin, J. Opt. Technol. 75, 636 (2008).

${ }^{23}$ A. Gorodetsky, I. T. Leite, N. Bazieva, and E. U. Rafailov, "Optical pump Terahertz probe carrier lifetime measurement in InAs/GaAs quantum dots based photoconductive antennae," in 2015 European Conference on Lasers and ElectroOptics - European Quantum Electronics Conference (Optical Society of America, 2015), p. CC_5_5.

${ }^{24}$ C. A. Schmuttenmaer, Chem. Rev. 104, 1759 (2004).

${ }^{25}$ J. E. Pedersen, V. G. Lyssenko, J. M. Hvam, P. U. Jepsen, S. R. Keiding, C. B. Sørensen, and P. E. Lindelof, Appl. Phys. Lett. 62, 1265 (1993).

${ }^{\mathbf{2 6}}$ R. Heitz, M. Veit, N. N. Ledentsov, A. Hoffmann, D. Bimberg, V. M. Ustinov, P. S. Kop'ev, and Z. I. Alferov, Phys. Rev. B 56, 10435 (1997).

${ }^{27}$ D. A. Yarotski, R. D. Averitt, N. Negre, S. A. Crooker, A. J. Taylor, G. P. Donati, A. Stintz, L. F. Lester, and K. J. Malloy, J. Opt. Soc. Am. B 19, 1480 (2002).

${ }^{\mathbf{2 8}}$ M. P. Lumb, E. Clarke, E. Harbord, P. Spencer, R. Murray, F. Masia, P. Borri, W. Langbein, C. G. Leburn, C. Jappy, N. K. Metzger, C. T. A. Brown, and W. Sibbett, Appl. Phys. Lett. 95, 041101 (2009).

${ }^{29}$ S. Gupta, M. Y. Frankel, J. A. Valdmanis, J. F. Whitaker, G. A. Mourou, F. W. Smith, and A. R. Calawa, Appl. Phys. Lett. 59, 3276 (1991).

${ }^{30}$ T. Müller, F. F. Schrey, G. Strasser, and K. Unterrainer, Appl. Phys. Lett. 83, 3572 (2003).

${ }^{31}$ D. Turchinovich, K. Pierz, and P. Uhd Jepsen, Phys. Status Solidi 1559, 1556 (2003).

${ }^{32}$ H. P. Porte, P. Uhd Jepsen, N. Daghestani, E. U. Rafailov, and D. Turchinovich, Appl. Phys. Lett. 94, 262104 (2009).

${ }^{33}$ E. A. Zibik, T. Grange, B. A. Carpenter, N. E. Porter, R. Ferreira, G. Bastard, D. Stehr, S. Winnerl, M. Helm, H. Y. Liu, M. S. Skolnick, and L. R. Wilson, Nat. Mater. 8, 803 (2009).

${ }^{34}$ I. T. Leite, A. Gorodetsky, R. Leyman, N. Bazieva, and E. U. Rafailov, "Towards high-intensity terahertz generation: InAs/GaAs quantum dots based antennae optically pumped up to 1 W," in 2015 European Conference on Lasers and Electro-Optics - European Quantum Electronics Conference (Optical Society of America, 2015), p. CC_5_4.

${ }^{35}$ N. Khiabani, Y. Huang, Y.-C. Shen, and S. Boyes, IEEE Trans. Antennas Propag. 61, 1538 (2013).

${ }^{36}$ N. Khiabani, Y. Huang, Y. Shen, and S. J. Boyes, IEEE Trans. Antennas Propag. 64, 2585 (2016). 